\title{
REVIEW
}

\section{The diagnosis, management and prevention of HIV-associated tuberculosis}

\author{
S Wasserman, ${ }^{1} \mathrm{MB}$ ChB, MMed, FCP (SA), Cert ID (SA) Phys; G Meintjes, ${ }^{1,2}$ MB ChB, FRCP (Glasg), FCP (SA), Dip HIV Man, MPH, PhD \\ ${ }^{1}$ Division of Infectious Diseases and HIV Medicine, Department of Medicine, Faculty of Health Sciences, University of Cape Town, South Africa \\ ${ }^{2}$ Clinical Infectious Disease Research Initiative, Institute of Infectious Disease and Molecular Medicine, Faculty of Health Sciences, \\ University of Cape Town, South Africa; and Department of Medicine, Imperial College London, UK
}

Corresponding author: S Wasserman (sean.wasserman@gmail.com)

\begin{abstract}
Tuberculosis (TB) and its strong association with HIV infection are the most important causes of the high rates of infectious morbidity and mortality in South African adults. The interaction between HIV and TB leads to more frequent smear-negative and extrapulmonary disease, resulting in atypical clinical presentations and altered performance characteristics of diagnostic tests. New and emerging diagnostics are being used to support earlier initiation of therapy and detection of drug resistance, although these have inherent limitations and empirical therapy is often still required. The management of HIV-associated TB is complicated by rapid clinical progression of disease, immune reconstitution inflammatory syndrome, drug-drug interactions and shared toxicities. A strong evidence base now provides guidance on the timing of initiation of antiretroviral therapy, the use of corticosteroids in TB and the use of isoniazid preventive therapy. This article provides a clinically oriented overview of the diagnosis, management and prevention of HIV-associated TB, with a focus on recent evidence in the field.
\end{abstract}

S Afr Med J 2014;104(12):886-893. DOI:10.7196/SAMJ.9090

The aim of this article is to update readers on recent evidence-based advances in the field and it is not intended to provide a comprehensive review of HIV-associated TB. Information was mainly sourced from personal archives and from citations in recently published reviews. Our references were selected based on relevance to the topic and impact on current practice. We have expressed the quality of evidence for each recommendation by describing the study methodology.

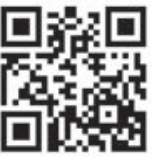

The colliding risk factors of HIV, poverty and a larg mining workforce have resulted in an unparalleled burden of tuberculosis (TB) in South Africa (SA) and neighbouring countries in terms of incidence rates. ${ }^{[1]}$ According to the most recent World Health Organization (WHO) global report, over half a million incident cases of TB occurred in SA in 2012 with an estimated $65 \%$ of these in HIV-co-infected patients. ${ }^{[2]}$ However, despite the availability of effective first-line antituberculous drugs and large-scale rollout of antiretroviral therapy (ART), TB is still the most common opportunistic disease and main driver of mortality in HIV, causing up to 100000 deaths in SA in 2012. ${ }^{[2]}$

Latent infection with $\mathrm{TB}$ has a much higher chance of reactivating in HIV as a result of loss of competent immune control, translating into an annual risk of up to $10 \%$, at least 12- to 20-fold higher than in immunocompetent individuals. ${ }^{[3]}$ Because TB causes higher rates of disease and mortality in HIV, ${ }^{[4]}$ it is a priority to make an early diagnosis, initiate antituberculous therapy timeously, provide appropriate HIV care (including ART and co-trimoxazole preventive therapy) and implement evidencebased interventions for prevention.

In this article we review the diagnosis, management and prevention of drug-susceptible TB in HIV-infected patients, with a focus on recent evidence. Tuberculosis-associated immune reconstitution inflammatory syndrome (TB-IRIS) has been reviewed in a recent $C M E$ article ${ }^{[5]}$ and a review of drug-resistant TB is included in this edition, so these topics will not be covered here. 
Table 1. Key performance characteristics of diagnostic tests for tuberculosis performed in HIV-infected patients

\begin{tabular}{|c|c|c|c|}
\hline Test & $\begin{array}{l}\text { Limit of detection } \\
\text { (bacilli/mL of } \\
\text { sputum) }\end{array}$ & Sensitivity & Comments \\
\hline Sputum culture (liquid system) & $10-100$ & $\begin{array}{l}80 \%{ }^{[11]} \text { (relative to clinical } \\
\text { reference standard) }\end{array}$ & $\begin{array}{l}\text { Reference test } \\
\text { Can be used for speciation and drug susceptibility } \\
\text { testing } \\
\text { Used to monitor response to therapy } \\
1-6 \text { weeks for result }\end{array}$ \\
\hline Sputum smear microscopy & $>10000$ & $30-40 \%^{[12-14]}$ & $\begin{array}{l}\text { Cannot distinguish non-tuberculous mycobacteria } \\
\text { from TB } \\
\text { Used to monitor response to therapy }\end{array}$ \\
\hline Sputum Xpert MTB/RIF & $10-100$ & $\begin{array}{l}\text { Single specimen } \\
\text { - Overall } 79-84 \%^{[15,16]} \\
\text { - Smear-negative disease } 68 \%^{[15]} \\
\text { (incremental yield from } 2 \text { and } 3 \text { specimens) }{ }^{[17]}\end{array}$ & $\begin{array}{l}\text { Rapid turnaround time } \\
\text { Detects rifampicin resistance } \\
\text { Cannot be used to monitor response to therapy } \\
\text { Positive results are not reliable after a recent } \\
\text { episode of TB treatment }\end{array}$ \\
\hline Determine TB-LAM Ag (urine) & NA & $\begin{array}{l}\text { Screening } 67 \%\left(\text { at } \mathrm{CD} 4^{+}\right. \\
\text {counts }<50)^{[18]} \\
\text { Inpatients } 40 \%{ }^{[19]}\end{array}$ & $\begin{array}{l}\text { High specificity } \\
\text { Best performance in } \mathrm{CD} 4^{+} \text {counts }<50 \\
\text { Very poor sensitivity with } \mathrm{CD} 4^{+} \text {counts }>100\end{array}$ \\
\hline
\end{tabular}

\section{Diagnosis}

The clinical presentation of HIV-associated $\mathrm{TB}$ is often nonspecific: cough is less frequent, ${ }^{[6]}$ and patients commonly present with extrapulmonary disease in the absence of respiratory symptoms and radiological abnormalities. ${ }^{[7]}$ The prevalence of smearnegative pulmonary $\mathrm{TB}$ is high, ranging from $25 \%$ to $61 \%,{ }^{[8]}$ and is associated with a poorer prognosis. ${ }^{[9-11]}$ It is therefore critical to understand how to select and interpret available diagnostics. These are discussed below and key performance characteristics are summarised in Table 1. ${ }^{[11-19]}$

\section{Supportive investigations}

Basic radiological and laboratory investigations are widely available and provide valuable information when assessing TB suspects with HIV. The drawbacks of these ancillary tests are limited sensitivity and specificity, but they have potential to support early empirical therapy when there is a high clinical pre-test probability of TB.
The chest X-ray (CXR) findings of $\mathrm{TB}$ in patients with $\mathrm{CD} 4^{+}<200$ cells $/ \mu \mathrm{L}$ are often atypical compared with the classic upperlobe cavitation seen in immunocompetent individuals, and may include nodular infiltrates, pleural effusions and intrathoracic lymphadenopathy. ${ }^{[20,21]}$ While these CXR patterns can be strongly supportive of $\mathrm{TB}$ in the correct clinical setting, up to $30 \%$ of patients with culture-confirmed HIV-associated pulmonary TB may have completely normal CXRs, ${ }^{[22]}$ limiting the sensitivity of this investigation.

Abdominal ultrasonography is useful as a rule-in test for HIV-infected TB suspects, especially in patients presenting with abdominal tenderness or otherwise unexplained systemic symptoms. ${ }^{[23]}$ Suggestive features include intra-abdominal lymphadenopathy, ascites, bowel-wall thickening and splenic hypodensities.

The finding of a lymphocyte-predominant exudate in pleural or pericardial fluid is strongly suggestive of TB in high-prevalence settings, and can often be used as a basis for empirical antituberculous therapy. But similarly to CXR and abdominal ultrasound, these findings are not specific for TB and may occur with other HIV-associated diseases such as cryptococcosis and lymphoma.

\section{Growth-based detection}

The reference standard for diagnosing TB is a positive culture of Mycobacterium tuberculosis on a clinical specimen. Culture remains the most sensitive method for diagnosis and allows for subsequent speciation, drug susceptibility testing and monitoring of microbiological response to therapy. Automated liquid culture systems have largely replaced solid media techniques because of simpler laboratory workflows, higher sensitivity and earlier detection times. However, the median time to detection still ranges between 11 and 15 days, ${ }^{[24,25]}$ and therefore cultures cannot be used to guide initial treatment decisions in a patient who is deteriorating rapidly. 
Growth of M. tuberculosis from a clinical site represents viable replicating bacilli, and should be interpreted as indicating active disease and the need for therapy. However, there is a substantial proportion of patients with culture-negative disease who respond to antituberculous therapy ${ }^{[1]}$ who should be evaluated according to the WHO 2007 clinical algorithm. ${ }^{[26]}$ This phenomenon may be related to an inadequate sample or a sample from an inappropriate clinical site being sent to the laboratory.

\section{Smear microscopy}

Sputum smear microscopy, traditionally performed with ZiehlNeelsen (ZN) staining, is a rapid, specific method of detecting infectious patients with $\mathrm{TB}$ and for monitoring microbiological response to therapy. Microscopy requires at least 10000 organisms per $\mathrm{mL}$ of sputum, and therefore will detect fewer cases in HIV-infected patients who frequently have paucibacillary or extrapulmonary disease. ${ }^{[6]}$ Yield can be increased by collecting two early morning specimens, but the gain in sensitivity with a third sputum specimen is very small, resulting in $2-5 \%$ more positives. ${ }^{[27}$ Sputum induction with nebulised hypertonic saline potentially identifies approximately $25 \%$ additional cases in symptomatic patients who are smear-negative or unable to spontaneously produce sputum. ${ }^{[28]}$

In some settings, over two-thirds of HIV-infected patients with active TB requiring therapy may be missed by smear microscopy. ${ }^{[12,29]}$ Although staining with auramine $\mathrm{O}$ and fluorescence microscopy improves sensitivity by about $10 \%,{ }^{[13]}$ overall performance remains suboptimal, meaning that a negative smear does not exclude the diagnosis of TB in HIV co-infected patients.

\section{Molecular methods}

Xpert MTB/RIF (Cepheid, Sunnyvale, CA, USA) is a closed real-time polymerase chain reaction (PCR) assay that amplifies an 81 base-pair region of the M. tuberculosis RNA polymerase $\beta$ subunit $(r p o B)$ gene. This target is specific for M. tuberculosis-complex and is the site of the majority of mutations associated with rifampicin resistance, therefore allowing rapid detection of rifampicin-resistant TB directly from clinical specimens.

Compared with culture as the reference test, the overall sensitivity of a single Xpert is $89 \%$, and about $10 \%$ lower in patients with HIV. As an add-on test after negative smear microscopy, the sensitivity drops to $67 \%,{ }^{[15]}$ thus still missing a third of sputum smear-negative culture-confirmed TB.

Because PCR is able to detect DNA within both live and dead organisms, molecular-based diagnostics may test positive despite being culture-negative. There are reports that the Xpert assay may remain positive after successful treatment of $\mathrm{TB},{ }^{[30]}$ and evidence that around a quarter of patients successfully treated for TB will be Xpert positive at 6 months. ${ }^{[31]}$ Therefore, Xpert should not be used to monitor response to therapy, and cannot
Table 2. Performance of Xpert for the diagnosis of extrapulmonary tuberculosis on selected specimens (compared against culture)

\begin{tabular}{llll}
\hline & Lymph node $^{[32]}$ & CSF $^{[32]}$ & Pleural fluid $^{[32]}$ \\
\hline Sensitivity, \% & 84.9 & 79.5 & 43.7 \\
Specificity, \% & 92.5 & 98.6 & 98.1 \\
CSF = cerebrospinal fluid. & &
\end{tabular}

be used alone to confirm a recurrent episode of $\mathrm{TB}$ after a recent treatment episode (the precise duration has not been defined but we suggest confirming with culture if treated for TB within the last year).

Xpert performs well on lymph-node aspirates, pus and cerebrospinal fluid (CSF), and the WHO recommends its use on these specimens as a replacement for smear microscopy for patients with suspected extrapulmonary tuberculosis (EPTB) or tuberculous meningitis (TBM). ${ }^{[32]}$ Clinicians should, however, be aware that Xpert still lacks sensitivity to exclude TBM in patients in whom the diagnosis is clinically suspected, and empirical therapy should not be withheld because of a negative CSF result. Xpert performs poorly with blood and pleural fluid, and is not recommended for use on these specimens (Table 2). There is emerging evidence that the assay has good performance characteristics on urine when used in patients with low $\mathrm{CD} 4^{+}$ counts and disseminated TB.

Other molecular diagnostic tests are available, including line probe assays (LPA) that use reverse hybridisation technology to speciate and detect drug resistance mutations in mycobacteria. Unlike Xpert, the LPA is prone to contamination and is less reliable when performed directly on clinical specimens. The value of LPA is its ability to detect resistance to other antituberculous drugs in addition to rifampicin, as well as detecting dual-strain infection. Its use on microscopypositive specimens or culture isolates has reduced time to initiation of multidrug-resistant TB therapy ${ }^{[33]}$

\section{Urinary lipoarabinomannan (LAM)}

LAM is a lipopolysaccharide molecule in the mycobacterial cell wall and serves as a virulence factor by promoting intracellular survival of M. tuberculosis. Detection of LAM in the urine of HIV-infected patients probably represents renal tract involvement with $\mathrm{TB} \cdot{ }^{[34]}$ The test is available as an immunochromatographic lateral flow assay or dipstick test (Determine TB-LAM Ag [urine LAM], Alere, Waltham, MA, USA) that can be used at point of care by adding unprocessed urine onto the test strip.

When LAM was used for screening outpatients entering an ART programme, the overall sensitivity of the urine LAM for diagnosing culture-confirmed TB was only $28.2 \%$, but was much 
higher (66.7\%) in the subgroup with $\mathrm{CD}^{+}$counts $<50$ cells/ $\mu \mathrm{L}$. When combined with smear microscopy the sensitivity rose further to $72.2 \%$, similar to a single-sputum Xpert. The specificity is excellent, exceeding 98\%, and the positive predictive value is over $90 \%$ in patients with advanced HIV. ${ }^{[18]}$ In another study, urine LAM provided a rapid diagnosis with a sensitivity of almost $40 \%$ among unselected HIV-infected medical admissions with a high prevalence of newly diagnosed EPTB. When urine LAM was combined with urine Xpert, TB was rapidly diagnosed in $85 \%$ of patients with $\mathrm{CD} 4^{+}$counts $<100$ cells $/ \mu \mathrm{L}^{\left[{ }^{[19]}\right.}$ Despite a growing evidence base, urine LAM has not yet been endorsed as a diagnostic test by the WHO and is currently considered to be investigational, but it may become available for clinical use in the future.

In summary, culture-based diagnostics remain the gold standard for microbiological diagnosis of TB. Smear microscopy is insensitive but important for identifying the most infectious patients and for monitoring response to therapy. Xpert represents a major advance in TB diagnostics with rapid identification of rifampicin resistance and a $30 \%$ higher yield than microscopy for pulmonary disease. ${ }^{[16]}$ However, it performs poorly for HIV-infected inpatients who are unable to produce sputum ${ }^{[19]}$ and cannot be used alone to diagnose recurrent episodes after recent treatment for TB. The use of Xpert on selected extrapulmonary specimens is now recommended by the $\mathrm{WHO}$ and will soon be introduced into local guidelines. Urine LAM is valuable for rapid point-of-care diagnosis, but should be reserved for admitted HIV-infected patients or those with $\mathrm{CD} 4^{+}$counts $<100$ cells $/ \mu \mathrm{L}$ as it performs poorly in other groups of patients.

\section{Management Approach}

TB results in more rapid clinical deterioration in HIV infection. Clinicians should have a high index of suspicion for TB in any HIV-infected patient with rapid loss of weight in the absence of diarrhoea, or with impairment in daily functions due to systemic symptoms, whether or not they have a cough. TB suspects with any of the following danger signs require hospitalisation for urgent investigation and consideration for empirical broad-spectrum antibiotics: unable to walk unaided, respiratory rate 30/minute, fever $39^{\circ} \mathrm{C}$ or pulse rate $120 /$ minute ${ }^{[26]}$ These patients should also be assessed for other life-threatening opportunistic infections such as Pneumocystis pneumonia, bacterial sepsis and cryptococcal disease with CXRs, blood cultures and serum cryptococcal antigen testing (if $\mathrm{CD}^{+}$counts $<100$ cells $/ \mu \mathrm{L}$ ). The decision to start empirical antituberculous therapy depends on the severity of the clinical presentation, response to initial antibiotics and access to diagnostic tests, with a low threshold to start early in ill patients or those with a rapidly deteriorating condition, even if the CXR is normal.
Potential pitfalls of empirical treatment include missing another serious opportunistic disease or drug-resistant TB and exposing a patient unnecessarily to a course of antituberculous therapy. Therefore, it is important to confirm the diagnosis with an appropriate culture-based or molecular assay. However, empirical therapy should not be delayed while awaiting results in patients with a high clinical pre-test probability of TB. Patients started on empirical therapy must be followed up closely and the diagnosis should be reconsidered if there is no clinical response after, or deterioration within, 2 - 4 weeks, especially if cultures are negative. An important cause of failure to respond to empirical antituberculous therapy is unrecognised drug-resistant $\mathrm{TB}$, further emphasising the need to collect adequate specimens for culture and drug susceptibility testing prior to initiating therapy.

\section{Antituberculous therapy}

The treatment of HIV-associated TB is the same as in HIV-negative patients. The WHO recommends a standard regimen of 2 months of rifampicin, isoniazid, ethambutol and pyrazinamide followed by 4 months of rifampicin and isoniazid, preferably in fixed-dose combination tablets and dosed according to weight. Therapy should be administered 7 days a week throughout the course. ${ }^{[35,36]}$

\section{Adjunctive therapy}

Unless contraindicated because of previous intolerance, all HIVinfected patients with TB should receive co-trimoxazole preventive therapy ( 2 single-strength tablets or 1 double-strength tablet daily) and pyridoxine (25 mg daily). Co-trimoxazole prophylaxis may prevent opportunistic infections such as Pneumocystis, isosporiasis and invasive Salmonella disease, and has been shown to reduce mortality by up to $46 \%$ in patients with HIV-associated TB. ${ }^{[37]}$ Although the optimal duration of therapy is unclear, it can be discontinued in nonmalaria endemic settings when the $\mathrm{CD}^{+}$count is $>200$ cells $/ \mu \mathrm{L}$. The recommendation for pyridoxine supplementation is based on the observation that sensory polyneuropathy is more common in patients with HIV and TB, possibly because of vitamin $\mathrm{B}_{6}$ deficiency caused by isoniazid therapy. ${ }^{[38]}$

Unless there is another compelling indication, the use of corticosteroids in HIV-associated TB is reserved for patients with significant symptoms related to TB-IRIS ${ }^{[39]}$ and for all cases of TBM. A randomised controlled trial (RCT) conducted in Vietnam demonstrated a $31 \%$ reduction in mortality in patients with TBM treated with high doses of steroids, regardless of severity of symptoms. There was also a trend towards improved survival in the subgroup of HIV-infected patients although this did not reach statistical significance. ${ }^{[40]}$ Prednisone is not recommended for tuberculous pericarditis in HIV, as it conferred no survival benefit and was associated with an increased risk of HIV-related malignancies in an RCT. ${ }^{[41]}$ 


\section{Antiretroviral therapy}

Observational studies have demonstrated a reduction in mortality risk of 64 - 95\% in patients with HIV-associated TB who are receiving concurrent $\mathrm{ART},{ }^{[42]}$ and an RCT performed in Durban showed improved survival for patients with $\mathrm{CD} 4^{+}$counts $\leq 500$ cells $/ \mu \mathrm{L}$ who started ART during antituberculous therapy ${ }^{[43]}$ Both WHO and the SA National Department of Health $(\mathrm{NDoH})$ guidelines recommend that all HIV-infected patients with active TB be initiated on ART regardless of $\mathrm{CD} 4^{+}$count.
The benefits of starting ART early in patients with TB need to be balanced against the risks of TB-IRIS and overlapping drug toxicities, which are more likely to occur if ART is started early after the start of TB therapy. Three RCTs have recently provided clarity on the issue of timing of ART in TB, all showing a survival benefit (or benefit in terms of a cumulative endpoint of mortality and AIDS progression) for patients with CD4 counts $<50$ cells $/ \mu \mathrm{L}$ starting ART after $\sim 2$ weeks of antituberculous therapy compared with deferring to around 8 weeks. ${ }^{[43-45]}$ Patients with $\mathrm{CD}^{+}$counts $>50$ cells $/ \mu \mathrm{L}$

Table 3. Common drug interactions between antituberculous therapy and ART

\begin{tabular}{|c|c|c|c|}
\hline Antituberculous therapy & Antiretroviral & Interaction & Comments \\
\hline \multirow[t]{8}{*}{ Rifampicin-based } & Efavirenz & Efavirenz concentrations not reduced & - No dose adjustment required \\
\hline & Nevirapine & Reduced concentrations of nevirapine & $\begin{array}{l}\text { - Preferably use efavirenz } \\
\text { - Omit } 200 \mathrm{mg} \text { daily lead-in dose and start with } \\
200 \mathrm{mg} \text { bd }\end{array}$ \\
\hline & Rilpivirine & Reduced concentrations of rilpivirine & - Do not co-administer \\
\hline & Etravirine & Reduced concentrations of etravirine & - Do not co-administer \\
\hline & Lopinavir/ ritonavir & Reduced concentrations of lopinavir & $\begin{array}{l}\text { - Requires double dose with } 4 \text { tablets }(800 / 200 \mathrm{mg} \text { ) bd } \\
\text { in adults (guidelines in children are different) } \\
\text { - Increase the dose gradually: } 3 \text { tabs bd for a week } \\
\text { then } 4 \text { tabs bd for duration of rifampicin use } \\
\text { - Reduce dose back to } 2 \text { tabs bd } 1 \text { - } 2 \text { weeks after } \\
\text { rifampicin stopped }\end{array}$ \\
\hline & $\begin{array}{l}\text { Atazanavir/ } \\
\text { ritonavir }\end{array}$ & Reduced concentrations of atazanavir & - Do not co-administer \\
\hline & $\begin{array}{l}\text { Darunavir/ } \\
\text { ritonavir }\end{array}$ & Reduced concentrations of darunavir & - Do not co-administer \\
\hline & Raltegravir & Reduced concentrations of raltegravir & $\begin{array}{l}\text { - A recent clinical trial suggests that dose increase } \\
\text { is not required }{ }^{[48]}\end{array}$ \\
\hline \multirow[t]{5}{*}{ Rifabutin-based } & Efavirenz & Reduced concentration of rifabutin & - Requires increased dose of rifabutin $450 \mathrm{mg}$ daily \\
\hline & Nevirapine & Non-significant increase in rifabutin & - No dose adjustment required \\
\hline & Rilpivirine & Reduced concentrations of rilpivirine & $\begin{array}{l}\text { - Avoid if possible, otherwise requires increased } \\
\text { dose of rilpivirine } 50 \mathrm{mg} \text { daily }\end{array}$ \\
\hline & Etravirine & Reduced concentrations of etravirine & $\begin{array}{l}\text { - Do not co-administer if used together with a } \\
\text { boosted protease inhibitor }\end{array}$ \\
\hline & $\begin{array}{l}\text { Ritonavir-boosted } \\
\text { protease inhibitors }\end{array}$ & Increased concentrations of rifabutin & $\begin{array}{l}\text { - Requires reduced dose of rifabutin } 150 \mathrm{mg} \\
\text { alternate days } \\
\text { - Can be used with standard doses of ritonavir- } \\
\text { boosted lopinavir, atazanavir and darunavir }\end{array}$ \\
\hline
\end{tabular}


do not have an increased risk of death or AIDS-defining events if ART is delayed for up to 8 weeks, thus providing an important opportunity to establish patients on antituberculous therapy and provide counselling for lifelong treatment before starting ART. Patients with all forms of TB and $\mathrm{CD} 4^{+}$counts $>50$ cells $/ \mu \mathrm{L}$ should therefore start ART within 8 weeks of TB treatment, and clinicians should ensure that they remain engaged in ART counselling until they start ART. However, it is advised that if these patients have other stage 4 defining conditions then ART should also be started at 2 weeks (except for cryptococcal meningitis, where ART should be deferred to 4 - 6 weeks after diagnosis). ART should be delayed for 4 - 8 weeks in patients with TBM regardless of $\mathrm{CD}^{+}$count. ${ }^{[46]}$

The WHO-recommended ART regimen for patients with TB is tenofovir, lamivudine/emtricitabine and efavirenz (EFV). These drugs are available in fixed-dose combination tablets and do not require dose adjustment when given with antituberculous therapy. Despite a US Food and Drug Administration (FDA) recommendation to use higher doses of EFV during treatment with rifampicin, there is no evidence that EFV concentrations are significantly lower or virological outcomes worse in patients being treated for TB and with standard EFV dose, and the practice of increasing EFV dose to $800 \mathrm{mg}$ daily is not advised.

Nevirapine (NVP)-based ART is inferior to EFV-based regimens in patients with TB. This was recently demonstrated in the CARINEMO trial, where fewer patients on NVP-based ART achieved HIV viral suppression compared with those taking EFV ${ }^{[47]}$ NVP also results in higher rates of cutaneous drug reactions and liver injury than EFV, and its use should therefore be limited to patients who are unable to tolerate EFV. If NVP is prescribed for patients with $\mathrm{TB}$, the starting dose should be $200 \mathrm{mg}$ twice daily (the lead-in dose of $200 \mathrm{mg}$ daily is omitted) because of enzyme induction by rifampicin.

\section{Drug toxicity and interactions}

There are multiple potential drug interactions and shared toxicities between ART and antituberculous therapy, especially when treating drug-resistant TB or using second-line ART. Tables 3 and 4 list the most clinically significant drug interactions and toxicities of commonly used agents, with advice on co-administration. There are also important potential interactions between antituberculous drugs and other commonly used drugs in HIV such as antifungals, macrolides and steroids, and it is important to check these before prescribing.

Cutaneous drug reactions (CDRs) are common in HIV-infected patients with $\mathrm{TB}$ and have a wide spectrum of clinical presentations. These range from mild morbiliform rashes to Stevens-Johnson syndrome or systemic involvement with eosinophilia and hepatitis. Mild rash can be treated symptomatically with continuation of all drugs and close clinical observation. Patients with severe CDRs need to immediately stop all potentially offending agents and be admitted to hospital. Co-trimoxazole is an important cause of adverse drug reactions in HIV and should be stopped in all cases of suspected drug rash. In general, non-nucleoside reverse transcriptase inhibitors (NNRTIs) should be avoided once implicated in a severe CDR, but it is often possible to successfully rechallenge antituberculous drugs. Expert advice should be sought in all cases.

The Southern African HIV Clinicians Society has recently published a comprehensive guideline on drug-induced liver injury (DILI) in HIV-associated TB. ${ }^{[49]}$ Table 5 lists important indications for stopping drugs implicated in CDRs and DILI.

\section{Isoniazid preventive therapy (IPT)}

IPT is established as a safe and effective measure to reduce TB in HIV-infected individuals, ${ }^{[50]}$ and has an additive effect when given

Table 4. Important shared toxicities

\begin{tabular}{|c|c|c|c|}
\hline Adverse effect & Antituberculous drug & Antiretroviral & Other \\
\hline Gastrointestinal disturbance & All TB drugs especially ethionamide and PAS & AZT, PIs, ddI & Macrolides \\
\hline Liver injury & $\begin{array}{l}\text { RIF, INH, PZA, quinolones, ethionamide, } \\
\text { PAS }\end{array}$ & NNRTIs, PIs & $\begin{array}{l}\text { Co-trimoxazole, azole } \\
\text { antifungals, anticonvulsants }\end{array}$ \\
\hline Nephrotoxicity & Injectables, RIF & $\mathrm{TDF}^{*}$ & Amphotericin B \\
\hline Drug rash & All TB drugs & NNRTIs (NVP > EFV), DRV, RAL & Co-trimoxazole \\
\hline Peripheral neuropathy & INH, terizidone, ${ }^{\dagger}$ ethionamide, linezolid & d4T, ddI & \\
\hline Neuropsychiatric & $\begin{array}{l}\text { INH (especially high dose), terizidone, } \\
\text { ethionamide }\end{array}$ & EFV & \\
\hline
\end{tabular}


together with ART. ${ }^{[51]}$ A meta-analysis of RCTs conducted in the pre-ART era demonstrated that IPT reduces the incidence of TB by $33 \%$ overall, with greater benefit (62\%) and a reduction in mortality in those who are tuberculin skin test (TST) positive. ${ }^{[50]}$ In a trial subsequently conducted in Botswana the risk of TB was reduced by $74 \%$ in TST-positive individuals with HIV who took IPT for 3 years compared with 6 months. ${ }^{[52]}$ Observational data from Soweto also suggest that continuous IPT is safe and effective. ${ }^{[53]}$ Yet despite this compelling evidence and inclusion in $\mathrm{NDoH}$ guidelines, uptake has been poor.

An ideal setting to upscale IPT is in ART clinics, where patients are already established in care and are more likely to adhere to IPT. ${ }^{[54]}$ With the implementation of a higher $\mathrm{CD} 4^{+}$count threshold for ART initiation in SA, more individuals will be attending ART clinics, further increasing the numbers that will potentially derive clinical benefit from this intervention. Findings from a recent RCT involving clinic patients on ART in Cape Town support this approach, where the reduction in incident $\mathrm{TB}$ was $37 \%$ in the group assigned IPT (versus placebo) for 12 months. Importantly, benefit was also seen in the subgroup of TST-negative patients, thus removing the requirement for TST testing prior to a 12-month course of IPT in patients on ART. ${ }^{[51]}$ This study has informed national guidelines, ${ }^{[55]}$ which now recommend 1 year of isoniazid $300 \mathrm{mg}$ daily (plus pyridoxine) for all HIV-infected patients on ART, regardless of TST status. In patients who are TST-positive this should be extended to 36 months or longer, based on the Botswana study findings. ${ }^{[52]}$

An evidence-based approach to IPT is presented in Fig. 1. Although the rates of isoniazid monoresistance in people who develop TB while on IPT are similar to those of controls, ${ }^{[56,57]}$ the

\section{Patient on ART}

\section{Patient not on ART}

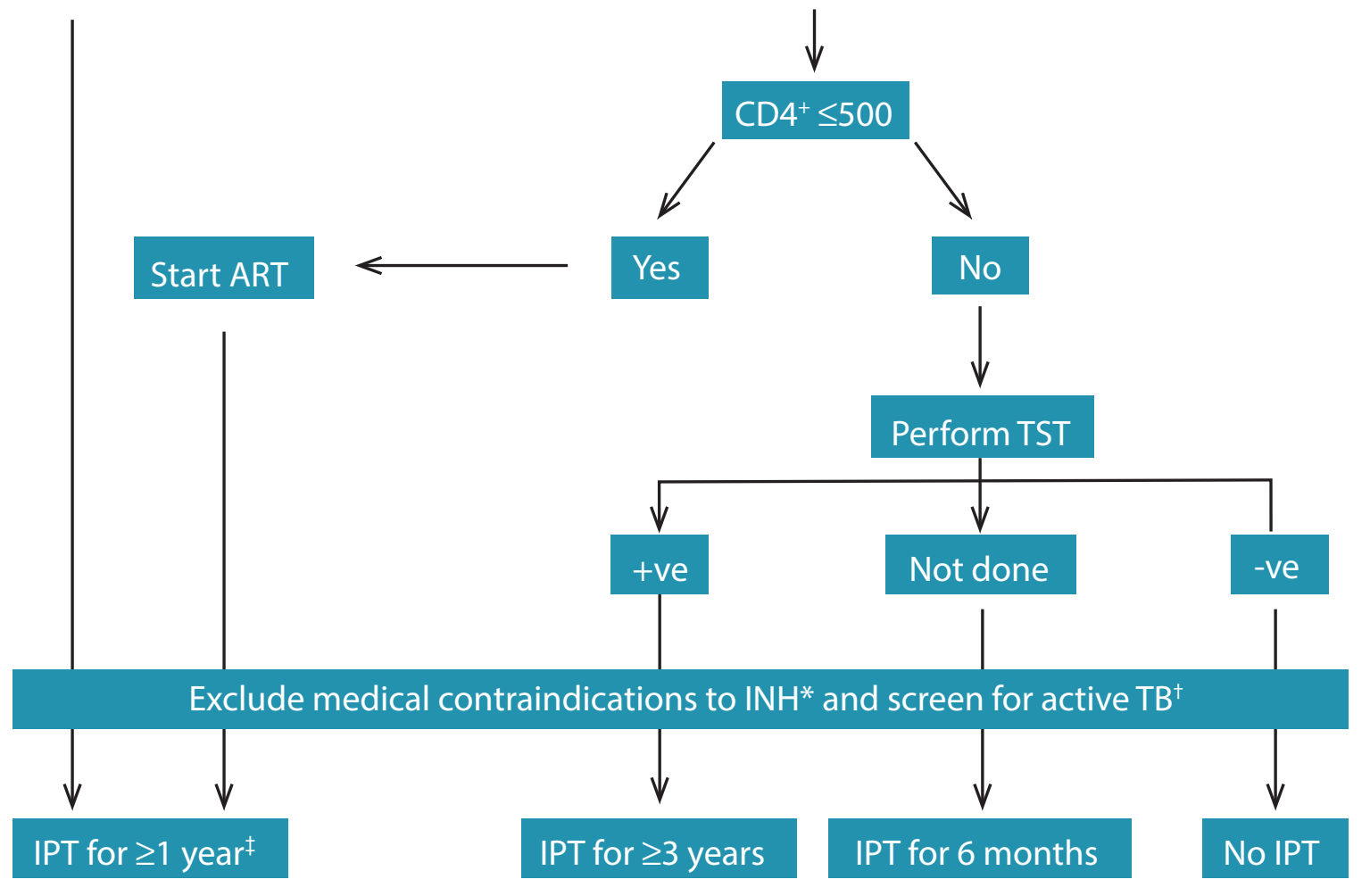

Fig. 1. Approach to prescribing isoniazid preventive therapy (IPT) (based on South African National Department of Health Guidelines ${ }^{[55]}$ and evidence from clinical trials $\left.{ }^{[50-52]}\right)$. "Hypersensitivity or intolerance to isoniazid, peripheral neuropathy, pre-existing liver disease or alcohol abuse. ${ }^{\dagger}$ WHO symptom screen: any cough, fever, night sweats or weight loss. If any symptom is present, defer IPT until confirmed culture-negative and symptoms have resolved. ${ }^{*}$ If TST is negative or not done, continue for 1 year; if TST is positive, continue for $\geq 3$ years. (INH = isoniazid; TST = tuberculin skin test.) 
Table 5. Indications for stopping suspected culprit drugs in CDRs and DILI

\begin{tabular}{ll}
\hline CDR & DILI \\
\hline Systemic symptoms & Symptomatic ${ }^{*}$ with ALT $>120$ IU/L \\
Mucosal involvement & Asymptomatic with ALT $>200$ IU $/ \mathrm{L}$ \\
Severe skin involvement & Any transaminitis with bilirubin $>40 \mu \mathrm{mol} / \mathrm{L}$ \\
Deranged liver enzymes & Prolonged INR or encephalopathy \\
CDR = cutaneous drug reaction; DILI = drug-induced liver injury; ALT = alanine transaminase; \\
$\begin{array}{l}\text { INR = international normalised ratio (prothrombin time). } \\
{ }^{*} \text { Nausea, vomiting, abdominal pain, jaundice. }\end{array}$
\end{tabular}

potential for this complication is greater if patients with active TB are inadvertently placed on isoniazid monotherapy. The WHO recommends using a four-symptom screen to exclude active TB prior to initiating IPT, ${ }^{[58]}$ and any patient with current cough, fever, night sweats or weight loss should not receive IPT until active TB is ruled out by a negative sputum culture and the symptoms have resolved.

\section{Conclusions}

TB is the most important cause of morbidity and mortality among HIV-infected people in SA. TB in HIV-infected patients poses complex clinical challenges because of atypical presentations, delayed diagnosis, more rapid clinical deterioration, drug interactions, TB-IRIS and overlapping toxicities. However, with the development of more rapid and accurate diagnostic tests, guidelines on empirical TB treatment, clear guidelines on initiation and timing of ART, an improved understanding of IRIS and adverse drug effects, and widespread implementation of IPT, we as clinicians can make an impact on the epidemic and its consequences.

References available online at http://dx.doi.org/10.7196/SAMJ.9090 